This item was submitted to Loughborough's Research Repository by the author.

Items in Figshare are protected by copyright, with all rights reserved, unless otherwise indicated.

\title{
Exploring the model development process in discrete-event simulation: insights from six expert modellers
}

PLEASE CITE THE PUBLISHED VERSION

http://dx.doi.org/10.1057/jors.2014.52

PUBLISHER

(C) Palgrave Macmillan

VERSION

AM (Accepted Manuscript)

LICENCE

CC BY-NC-ND 4.0

REPOSITORY RECORD

Tako, Antuela A.. 2019. "Exploring the Model Development Process in Discrete-event Simulation: Insights from Six Expert Modellers”. figshare. https://hdl.handle.net/2134/14457. 
This item was submitted to Loughborough's Institutional Repository (https://dspace.lboro.ac.uk/) by the author and is made available under the following Creative Commons Licence conditions.

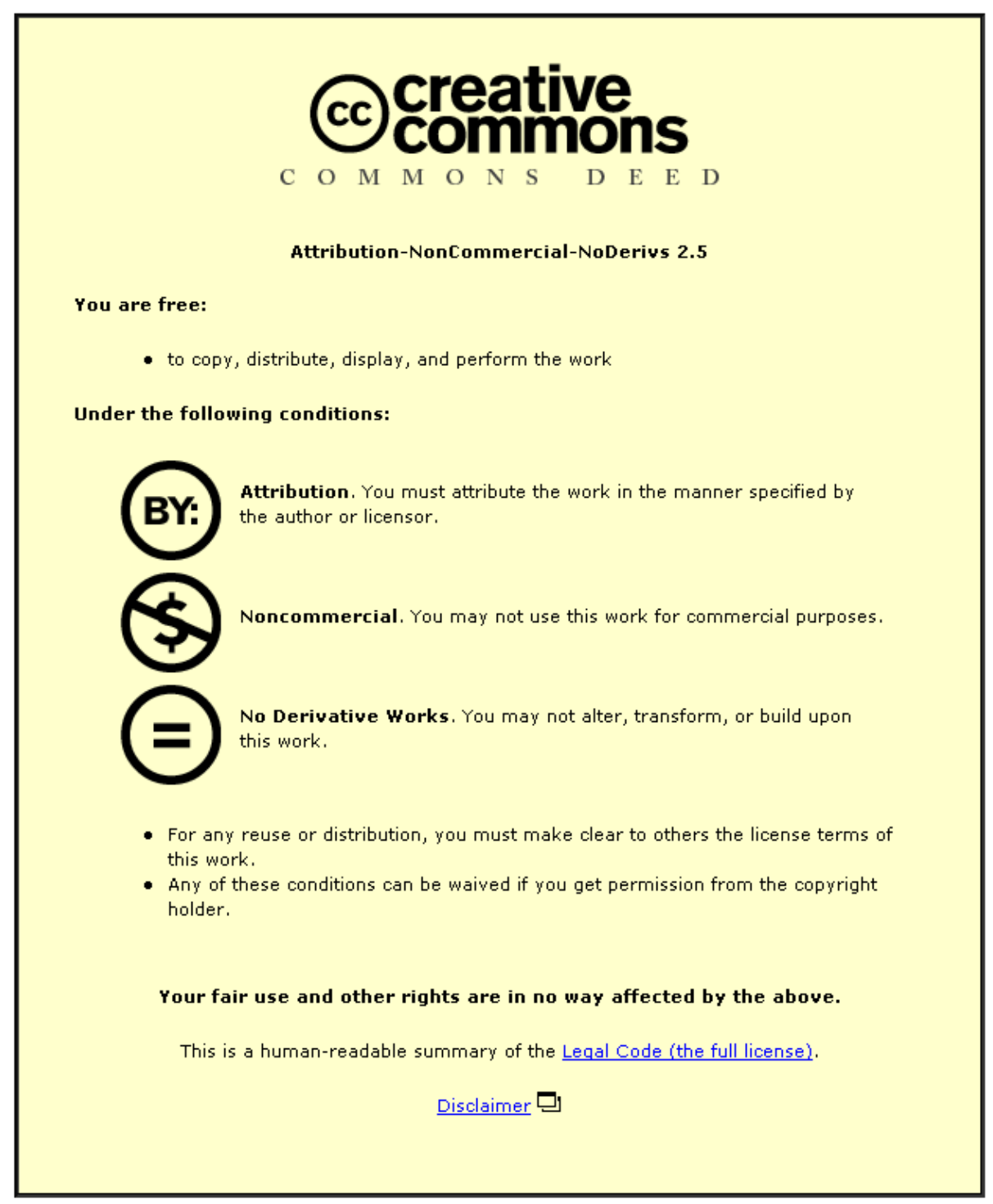

For the full text of this licence, please go to: http://creativecommons.org/licenses/by-nc-nd/2.5/ 


\title{
Exploring the model development process in discrete- event simulation: Insights from six expert modellers
}

\begin{abstract}
This paper explores the model development process in discrete-event simulation (DES) by reporting on an empirical study that follows six expert modellers while building simulation models. DES is a widely used modelling approach, however little is known about the modelling processes and methodology adopted by modellers in practice. Verbal Protocol Analysis is used to collect data, where the participants are asked to speak aloud while modelling. The results show that the expert modellers spend a significant amount of time on model coding, verification \& validation and data inputs. The modellers iterate often between modelling activities. Patterns of modelling behaviour are identified, suggesting that the modellers adopt distinct modelling styles. This study is useful in that it provides an empirical view of existing DES modelling practice, which in turn can inform existing research and simulation practice as well as teaching of DES modelling to novices.
\end{abstract}

Keywords: Simulation, model development, discrete-event simulation, modelling practice, education, verbal protocol analysis.

\section{Introduction}

In the last decades simulation has been adopted and used on a wider scale mainly due to developments in computing and the availability of simulation software (Nance and Sargent 2002; Robinson 2005). As a result, modellers with varying levels of modelling experience and skills have gained a wider access to simulation modelling. This has raised concerns about the misuse of OR (including simulation) models (Pidd 1999; Hollocks 2001; Robinson 2005). On the other hand, simulation modelling has been characterised as both an art and science (Shannon 1975; Shannon 1998). Moreover, while simulation research is rich in applications of simulation in business and industry, research on the simulation practice and methodology is limited. Studies in simulation 
methodology aiming to understand existing modelling processes are considered useful as they can help improve existing simulation practice in the field (Pidd 1999).

Experimental studies exploring how experts (mainly practitioners) build models in action are of special interest as they are based on real life practice as opposed to reporting opinions about the modelling practice followed. This paper is particularly interested in the model development process in discrete-event simulation (DES), which so far has not been considered in depth by existing experimental studies. Three main experimental studies are found in the literature. Willemain (1995) was the first to study how experts formulate OR models, particularly mathematical ones. Wang and Brooks (2007) undertook a similar study involving one expert (academic) and eighteen novice simulation modellers working on simulation projects. Both studies, Willemain (1995) and Wang and Brooks (2007) focus only on conceptual modelling or early stages of model development. Tako and Robinson (2010) observed expert modellers building simulation models on the computer to compare the model development process in discrete event simulation and system dynamics modelling. The current study expands on the work by Tako and Robinson (2010) to explore in more detail the whole model development process in DES. Further to conceptual modelling, this paper also explores model building on the computer. It expands on the data from previous research undertaken by Tako and Robinson (2010) to study in more detail the model development process of six DES experts (practitioners), while building simulation models on the computer. The analysis is furthermore extended with the identification of emerging patterns and modelling styles adopted during the modelling activity. The paper focuses specifically on one modelling approach, that of DES. It is expected that modelling using DES is different from other OR approaches based on findings of previous research by Tako and Robinson (2010) that suggest that DES and SD modellers follow a different approach to modelling.

The findings of this study inform the existing research and practice in DES by providing a better understanding of existing model development processes. Its contribution is threefold. Firstly, it informs DES model- 
ling methodology theory and academic research on the main characteristics of the modelling process as observed in experts' modelling activities. Secondly, the insights gained can enhance simulation modelling practice, by raising awareness among practitioners and researchers of the processes involved and potential improvements. Last but not least, it can help improve modelling skills and teaching of DES modelling to novices.

The rest of this paper is organised as follows. Section 2 explores existing empirical work studying the DES model development process, followed by section 3 where the study, including the case study, the participants, the research method used, and the Verbal Protocol Analysis (VPA) are described. The quantitative analysis undertaken and the results obtained are provided in section 4 . Section 5 discusses the results of the study, limitations and recommendations for further work. Finally, section 6 concludes the paper with considerations about the practical implications of the findings of the study.

\section{Existing work on model development methodology}

In this section, existing work on simulation modelling methodology is considered. The studies reviewed consist of three types, the ones providing advice and guidelines, surveys interviewing simulation modellers and experimental studies.

A number of authors, usually seasoned modellers in DES, describe the modelling process in what they often call simply models or life cycle models providing also guidelines for modellers to follow through the steps prescribed (Balci and Nance 1987; Nance 1994; Pidd 2004; Robinson 2004; Sargent et al. 2006; Law 2007; Sargent 2010; Balci 2012). Others provide advice on common pitfalls and modelling principles for others to follow based on their wide experience as modellers (Shannon 1998; Pidd 1999; Sanchez 2007). Both Pidd (1999) and Morris (1967) emphasize the importance of learning the modelling skills as compared to learning about models. They both put forward modelling principles, the latter calls them hypotheses, to assist novice modellers in their modelling journey. Among others, both authors suggest starting out with small models and then 
adding detail gradually. They also recommend that complex problems can be divided into smaller models/ components. In addition, Shannon (1998) lays out the "40-20-40 Rule", referring to the proportion of time spent among 12 modelling activities defined. According to Shannon, 40\% of the effort and time of a project should be devoted to problem definition, project planning, conceptual modelling and data collection, $20 \%$ to model coding and the remaining $40 \%$ to verification and validation, experimentation and reporting. Almost every author emphasises the iterative nature of the modelling process (Morris 1967; Balci 1994; Pidd 1999; Banks et al. 2001; Robinson 2004).

Another type of existing work includes studies that undertake surveys with simulation practitioners. For example, Cochran et al. (1995) surveyed the effort spent among different activities during a simulation project. He found that data collection was the most time-consuming activity, acquiring almost $15 \%$ of total project effort, followed by problem formulation, model translation (equivalent to conceptual modelling) and model building. In another study, Hollocks (2001) asked four modellers working for major organizations to estimate the amount of time spent among modelling activities. He reports that approximately $40 \%$ of the time is spent on modelling, $20-30 \%$ on experimentation and $30-40 \%$ on pre-modelling and post-experimentation. Foss et al. (1998) interviewed expert modellers on their practice of developing simulation models. Their results suggest that modelling is a creative activity dependent on personal style, background and characteristics of the modeller. They also found that the more experienced modellers tend to undertake less conceptual modelling and that the diagramming method used is affected by modellers' background.

Other studies undertake interviews to define simulation project success and to identify influencing factors (Robinson and Pidd 1998; McHaney et al., 2002). Robinson and Pidd (1998) developed a four stage model of simulation project success, which suggests that the perception of success for simulation providers (modellers) and their customers can vary throughout the life of a project. This study suggests that success boils down to the careful management of expectations between the two parties involved. McHaney et al (2002) surveyed 
simulation practitioners and found among others that effective communication of outputs, higher levels of analyst experience and structured approaches to model development characterise simulation project success.

Willemain was the first to undertake an experimental study to explore model formulation in OR, as followed by expert modellers (Willemain 1994; Willemain 1995). The study involved mainly mathematical models and focused primarily on the early stages of model development. The same approach was later used to compare the initial findings to the modelling process followed by novice modellers in OR (Powell and Willemain 2007; Willemain and Powell 2007) as well as to study the use of sketches by expert OR modellers (Waisel et al. 2008).

Wang and Brooks (2007) undertake an experimental approach with the view to understanding conceptual modelling processes in DES as followed by novices (eighteen students) and one expert. Participants were asked to build simulation models of real (not pre-defined) problems. The data were collected by the participants, who were asked to record the time spent on the different modelling activities as they carry out the simulation projects. Even though the study focuses on the early stages of model development it reports on the amount of time spent among the different modelling activities by the expert modeller and novice modellers. The activities undertaken by the expert modeller are approximated to match the novice modellers' reports and compared to find that the expert modeller attaches more importance to conceptual modelling and verification and validation, whereas novice modellers focus more on data collection and report writing. Albeit this study provides useful insights about the DES modelling process, it focuses on the early stages of model development and it is furthermore based on the activities followed by one expert modeller. The data collected was based on the participant's reports, lacking the level of detail obtained when the process is observed first-hand by the researcher. 
Tako and Robinson (2010) compare the model development process between two groups of expert modellers involved (five discrete-event simulation and five system dynamics modellers). Verbal Protocol Analysis (VPA) is employed to record the expert modellers' thinking process as they undertake an experimental exercise, which involves building simulation models of the UK prison population. The study considers the model development processes with the view to comparing the two simulation approaches, while a detailed analysis of the respective modelling processes is not undertaken. The data from the latter study is further extended in the current paper to explore in more detail the model development process in DES modelling.

Summarizing on the literature considered in this section, a number of authors provide guidelines in relation to simulation modelling methodology, but there are limited studies that explore the simulation model development process in action. Existing studies following modellers while formulating or developing models such as those undertaken by Willemain $(1994 ; 1995)$ and (Powell and Willemain 2007; Willemain and Powell 2007) and those by Wang and Brooks (2007) and Tako and Robinson (2010), do not focus specifically on the DES model development process. Extending on existing studies, the current paper explores the DES model development process, by observing six expert modellers and their thought processes while building models in a controlled laboratory setting. The overall objective of this study is to explore the model development process in discrete-event simulation (DES), based on the modelling activities attended to and pattern of iterations observed for each individual expert. The process is expected to be iterative as already found by previous studies in general OR (Willemain 1995) and simulation (Wang and Brooks 2007; Tako and Robinson 2010) modelling.

\section{Methods}

In this section the study undertaken is explained. First, the modelling exercise is briefly described, followed by an introduction to the research method used that of verbal protocol analysis (VPA). Next, the profile of the participants involved in the study is explained and the coding process carried out. 


\subsection{The modelling exercise}

The modelling exercise used as the stimulus for inducing modellers' thoughts asked expert modellers to build models of the UK prison population, based on a study reported by Grove et al. (1998). The UK prison population problem was considered suitable because it is sufficiently self-contained to enable the development of a simulation model in a limited time period of around 60 minutes. The time limitation was necessary to ensure participation of simulation practitioners with busy time schedules. The topic is furthermore a topical problem in the UK, often receiving media coverage, hence it is reasonably familiar to most participating simulation experts. Furthermore, this case study has been already used to compare the development and use of models in discrete-event simulation and system dynamics (Tako 2009; Tako and Robinson 2009; Tako and Robinson 2010).

The case study provides a brief introduction to the prison population problem with particular attention to the issue of overcrowded prisons. Descriptions of the reasons for, and impacts of, the problem were also provided, including figures and facts, most of which are based on reality, but slightly adapted for the purposes of the study. Two types of prisoners are considered, petty and serious offenders. There is already an initial number of prisoners in the system $(76,000)$. Offenders enter the system as first time offenders and receive a sentence depending on the type of offence. Petty offenders enter the system at a higher rate, on average 3,000 people/year vs. 650 people/year for serious offenders, but receive a shorter sentence length, on average 5 years vs. 20 years for serious offenders. After serving time in prison the offenders are released. A proportion of the released prisoners re-offend and go back to jail (recidivists) after on average 2 years. Petty prisoners are more likely to re-offend.

Participating modellers were asked to build a simulation model to be used as a decision support tool for policy making. Two possible scenarios were provided, the increase of the current prison capacity and so facilitate the introduction of stiffer rules, or alternatively to reduce the size of the prison population by introducing 
alternatives to jail and/or enhancing the social support provided to prisoners. For more details on the case study the reader is referred to Tako (2009). For interested readers, the case study is available by the author upon request.

\subsection{Verbal Protocol Analysis (VPA)}

VPA is a research method derived from psychology which requires the subjects to 'think aloud' when making decisions or judgments while exposed to a common stimulus. It relies on the participants' generated verbal protocols in order to understand in detail the mechanisms and the internal structure of cognitive processes that take place (Ericsson and Simon 1984). Therefore, VPA as a process tracing method provides access to the activities that occur between the onset of a stimulus (reading the case study) and the eventual response to it, in this case building a representative model (Ericsson and Simon 1984; Todd and Benbasat 1987). Willemain $(1994 ; 1995)$ was the first to use it in Operational Research (OR) to document the thought processes of OR experts while formulating models.

VPA is considered to be an effective method to study the model development process in simulation modelling. It is useful because of the richness of information and the live accounts it provides on the experts' modelling process. It furthermore overcomes problems encountered with alternative methods such as interviews or questionnaire surveys, where data collected rely on respondents' reflections on their modelling which may not reflect practice truthfully. This can be either because they rely on respondents' memory of how they go about modelling or due to the desire of conforming to the generally accepted rules. In the current study, VPA is used to capture modellers' thoughts in practical modelling sessions in a controlled experimental environment, using a common stimulus, that is building a model of the UK prison population.

Protocol analysis, however has its own limitations. The verbal reports may omit important data (Willemain 1995) because the experts being under observation may not behave as they normally would. The participating 
modellers are asked to work alone and this way of modelling may not reflect their usual modelling practice, where they would interact with the client or other team members. In addition, there is the risk that participants do not 'verbalize' their actual thoughts, but are only 'explaining'. To overcome these limitations and to encourage participants to speak their thoughts aloud, short verbalization exercises between one to three minutes long were administered at the beginning of each session. Three out of four verbalisation exercises (a multiplication exercise, solving an anagram, counting the number of windows in one's parents' house, finding one's position in an imaginary ordered queue of four), based on Ericsson and Simon (1984), were chosen at random for each participant.

\subsection{The modelling sessions}

The expert modellers involved in this study were provided with the prison population case study, which they read in silence at the start of the session and then they were asked to proceed with building a simulation model using their preferred simulation software. During the modelling process experts were asked to 'think aloud' while modelling for recording purposes. The researcher sat in the same room, but social interaction was limited. She only intervened when participants stopped talking for more than 20 seconds to encourage them to "keep talking". Explanatory questions were also answered. The modelling sessions were held separately with each individual modeller, in an office environment. The sessions lasted approximately 60-90 minutes, which were recorded and afterwards transcribed. The participants had access to writing paper and a computer with relevant simulation software. It should be noted that due to the limited time available, participants were asked to build "rough" models as opposed to fully complete models deliverable to the client.

\subsection{The Participants}

Six practitioners, experts in DES modelling took part in this study. For the purposes of this study, an expert modeller is considered a practitioner, who has at least 4 years' experience and uses simulation modelling as part of their work. Involving practitioners, who apply simulation modelling mainly in consultancy projects, ra- 
ther than academics, is considered appropriate given that this research is concerned with the practical aspect of simulation modelling as opposed to the theory involved. The sample size of six practitioners is considered reasonable for a VPA study, given the richness of data found in one protocol. According to Todd and Benbasat (1987), VPA samples tend to be small, between two to twenty.

For reasons of confidentiality participants' names are not revealed, instead each participant is given a number, hence called modeller 1, 2, 3, etc. They all hold senior consultant positions in established software and/or consultancy companies in the UK. Table 1 provides a list of participants' profiles, including modelling experience, simulation software used in the modelling sessions, source of obtaining simulation skills as well as their education level and degree title.

\section{TABLE 1 ABOUT HERE}

The participants' experience in simulation modelling ranges between 4 and 13 years. They boast an extensive experience of modelling in fields such as: health care, criminal justice, food \& drink, supply chain, etc. The modellers were asked to use their preferred simulation software in the modelling sessions. As a result three participants used Witness, two Simul8 and only one Flexsim. This provides some level of diversity among the participants in terms of length of modelling experience and simulation software used. The six expert modellers had a mixture of backgrounds, having completed undergraduate or masters' degrees in Engineering, Computer Science or Operational Research. They obtained simulation modelling skills either as part of their job or through a combination of studies and job.

\subsection{Coding the Protocols}

A coding scheme was devised in order to identify what the modellers were thinking about in the context of simulation modelling. The coding scheme was devised following the generic activities undertaken in typical DES simulation projects, based on the existing DES literature ( Shannon 1975; Banks et al. 2001; Pidd 2004; 
Robinson 2004; Law 2007). The modelling topics defined are based on the broad categorization of DES modelling activities to include: problem structuring, conceptual modelling, data collection and analysis, model coding, experimentation, implementation and verification and validation. A definition of each modelling topic is next provided in the list below:

1. Problem structuring (PS): References to the problem and the issues (objectives) to be addressed by the project or model.

2. Conceptual modelling (CM): References to different parts of the model, drawings of a conceptual diagram $r$, , specification of assumptions and simplifications, consideration of ways to represent the large number of people (prisoners) in the system, definition of variables to be included and/or varied in the model.

3. Model coding (MC): References to the types of entities, i.e. activity (Simul8) or processes (Witness) entered, addition of qualifiers such as labels, rules (push or pull in Witness), arrows (in Simul8), definition of the units used to represent time or people, modelling the initial conditions of the system, development of a user interface, documentation with explanations of the entities modelled.

4. Data inputs (DI): References to the already provided data, specific distributions and their parameters and/or to the need for additional data or parameters.

5. Model results \& experimentation (EXP): References to model outcomes(i.e. key performance indicators ), consideration of the different scenarios or of variables varied for experimentation. .

6. Implementation (IMP): References to the implications on the society based on the findings of the model, consideration of how the findings could be used. 
7. Verification \&Validation (V\&V): References to checking that the model works as intended, checking that the results are correct, identifying the reasons for which the model is stuck or not working properly, considerations about testing the model with the client.

After each modelling session was completed the audio-recorded verbal protocol was transcribed. Each transcript, consisting of one verbal protocol was next divided into episodes or 'thought' fragments, where each fragment is the smallest unit of data meaningful to the context of this research. The length of each episode varies depending on the modelling aspect the modeller considers during the modelling process. Then each episode was coded into one of the 7 modelling topics or an 'other' category for verbalizations that were not related to the modelling task. Some episodes referred simultaneously to 2 modelling topics and, therefore, were coded using two modelling topics. The same coding scheme as in (Tako and Robinson 2010) was used, where also a pre-test with 4 academics was initially undertaken to test the codes. Two example extracts follow:

"The sentence length for petty offenders is 5 years and the sentence length for serious is 20 years. OK." DES2, episode 3 (Data Inputs)

"Looking at two sets of parts coming in with different variables and how long they stay in prison and at the end of the time then I'll have a ...[inaudible] when they get released out of the prison buffer, have a time, so a percentage will go into the prison buffer for petty and serious, and after the percentage that goes to the buffer they will have some time before they go back into prison again." DES1, episode 5 (Conceptual Modelling)

The transcripts were coded manually using a standard word processor. According to Willemain (1995), the coding process requires attention to the context a phrase is used in and, therefore, subjectivity in the interpretation of the scripts is unavoidable. In order to deal with subjectivity, multiple independent codings were undertaken in two phases. The transcripts were initially coded twice with a gap of three months between cod- 
ings. Overall, a 93\% agreement between the two sets of coding was achieved, which was considered acceptable. The differences were examined and a combined coding was reached. Next, the coded transcripts with the combined codes were further blind checked by a third party, knowledgeable in simulation modelling. Overall, a $90 \%$ agreement was achieved. The differences were discussed and re-examined by both parties to arrive at a consensus coding. The results from the coded protocols are next presented and discussed.

\section{Results}

This section presents the results of the analysis undertaken on the six verbal protocols collected, with a view to providing an account of the experts' modelling behaviour. The analysis explores three main aspects, the distribution of attention among modelling topics, the sequence of modelling topics attended to during the modelling exercise and the pattern of iterations followed among topics. The findings for each part of the analysis follow.

\subsection{Attention paid to modelling topics}

The number of words articulated by the six experts is used to measure the amount of verbalizations. The distribution of attention paid among the seven modelling topics is found by dividing the number of words for each topic by the total number of words verbalised. Figure 1 displays the proportion of the total words verbalized under each modelling topic by all six expert modellers. This shows that participating experts concentrate their attention mostly on model coding, verification \& validation and data inputs, by devoting $42 \%, 21 \%$ and $16 \%$ of their attention respectively. The attention paid to experimentation is lower. A more detailed analysis of the episodes on experimentation shows that modellers' attention concentrates mainly on exploring model results rather than on setting up alternative scenarios. The amount of verbalizations for problem structuring and implementation is also limited.

FIGURE 1 ABOUT HERE 
Considering the above proportions against Shannon's (1998) 40-20-40 rule, some deviation can be observed. Problem structuring (PS), conceptual modelling (CM) and data inputs (DI) combined account for $30 \%$ of the attention compared to Shannon's recommended 40\%; model coding (MC) accounts for $42 \%$ against Shannon's recommended $20 \%$, whereas verification and validation (V\&V), results and experimentation (EXP) and Implementation (IMP) combined account for $25 \%$ against Shannon's recommended $40 \%$. It seems that the six experts paid more attention to $M C$ compared to $V \& V$ and experimentation. This divergence could be due to the nature of the task, which involved building DES models in a short period of time (one hour) in a controlled laboratory setting. On the other hand Shannon's rule is an anecdotal estimation, which is not based on empirical evidence.

Comparing now these results to those from the one expert modeller followed in Wang and Brooks' (2007) study more distinguished differences can be observed specifically for the attention paid to problem structuring, conceptual modelling and model coding (Table 2). Significant negative values of differences show that the six expert modellers in the current study paid less attention to problem structuring and conceptual modelling, whereas the high positive difference for model coding shows that they pay more attention to model coding compared to the expert modeller data in Wang and Brooks' (2007) study. These differences could be due to the different nature of the two studies; in the current study experts were provided with a relatively prestructured exercise, hence the modellers needed to think less about what needed to be modelled and were not required to prepare a report, whereas in Wang and Brook's (2007) study, a real life simulation project was followed. The latter study used a different data collection method, where the expert modeller was asked to record the time spent on the different activities himself/herself on a weekly basis. Furthermore, the expert modeller was an academic. For this reason the data from these two studies may not be directly comparable.

\section{TABLE 2 ABOUT HERE}


Figure 2 maps out the proportion of words articulated by each modeller for the 4 most frequently attended to modelling topics (CM, MC, DI, V\&V) in radar diagrams. Each modeller is represented in a different shade. As already seen from the proportion of attention paid to different modelling topics above, all modellers spend most of their time on model coding, which results in a skewed diamond-shape towards the model coding axis. For the rest of the modelling topics, even though the differences are relatively small, different modellers seem to exhibit a different pattern of attention as observed by the resulting shape of the radar diagrams.

\section{FIGURE 2 GOES ABOUT HERE}

Based on observations of the shape of the radar diagrams obtained three different patterns of modelling activity can be tentatively identified. The most skewed shapes representing the activity of modellers 1 and 5 , seem to exhibit an activity oriented towards model coding (Figure 2, b). The only characteristic these modellers have in common is that they used the same software (Witness) in their individual sessions. However, modeller 6 , who also used Witness does not fit this grouping. Considering the shapes of the radar diagrams for modellers 3 and 6 (Figure 2, c), these are less skewed towards model coding. A more symmetrically divided modelling activity is observed among the four modelling topics for these modellers, as they both pay reasonably similar attention to conceptual modelling, data inputs, V\&V and model coding, albeit the latter is slightly higher. Considering the modellers' profiles (Table 1), these two modellers can be classified as highly experienced. However, modellers 1 and 4, with an equivalent length of experience, do not exhibit a similar shape. The diamond shape for modellers 2 and 4 shows a modelling activity shared reasonably equally among model coding, data inputs and V\&V, but comparatively less attention is paid to conceptual modelling (Figure 2, d). The only common characteristic for modellers 2 and 4 is that they used the same software (Simul8) to build models.

Based on the above observations and considering the expert modellers' profiles (i.e. length of experience, education or software used), there are no clear distinguishing criteria to match the modelling patterns observed. 
Some of the styles seem to be related to the software used, however this does not seem to be representative of all modellers using the same software. At a coarse level, this could suggest that the different modelling activity exhibited is mainly due to the participants' modelling styles. Willemain (1995) collected a larger number of protocols and undertook multidimensional analysis on the distribution of attention to the modelling topics attended to by 24 expert OR modellers. He concluded that it is plausible to refer to the notion of 'individual modelling styles'. Based on the exhibited modelling activity of the participating experts, the three emerging modelling styles could be named as model coding-oriented (Figure 2, b), balanced style (Figure 2, c) and modelling with reduced conceptualisation (Figure 2, d). The author however acknowledges that the differences are relatively small hence the conclusions drawn about the different styles should be only considered tentative. Furthermore, it should born in mind that these modelling styles are subjectively identified based on the observed differences between the shape of the radar diagrams.

\subsection{Sequence of modelling topics}

Considering now the progression of the expert modellers' thinking over the time of undertaking the exercise, timeline plots are drawn for each individual modeller (Figure 3). The timeline plots are divided into the seven sets of plots for each modelling topic, where a bar appears whenever a topic is mentioned throughout the length of the protocol. The vertical axis takes three values, 1 when the specific modelling topic is attended to by the modeller, 0.5 when the modelling topic and another have been attended to at the same time and 0 when the modelling topic is not mentioned. The horizontal axis represents the proportion of the verbal protocol, from $0 \%$ to $100 \%$ of the number of words. The proportion of the verbal protocol is counted as the fraction of the cumulative number of words for each consecutive episode over the total number of words in that protocol, expressed as a percentage.

\section{FIGURE 3 GOES ABOUT HERE}


An overall observation of the timeline plots suggests that the modellers attend to different modelling topics at different times during the modelling exercise. All timeline plots seem to show that the modellers switch their attention frequently among topics. Next, each modelling topic and their sequence of occurrence is considered in more detail for the six modellers.

Even though Problem structuring was attended to by only three modellers (modellers 1, 3 and 5), it seems to be attended to at the beginning of the protocols, with the exception of modeller 5 who also attended towards the end of the protocol.

Attention to conceptual modelling appears at various points throughout the modelling task. Modellers 3 and 6 seem to have the highest density of bars, concentrated mostly at the beginning of the protocol. For modellers 1, 2, 4 and 5 the bars seem to be less dense and more spread across the protocol.

Model coding seems to be attended to throughout the modelling exercise by all six modellers. Most modellers delay model coding till after the first 10-15 minutes, but modellers 2 and 4 attend to model coding from the beginning of the modelling exercise. A high density of model coding bars can be observed throughout the protocols. This is expected based on the results discussed in section 4.1 above.

Data inputs also seems to be well attended to by most modellers. Some (modellers 1, 2 and 5) appear to enter the data into the model mostly during the first half, while others (modellers 3,4 and 6 ) throughout the modelling task.

Verification \& validation of the model seems to be attended to after some part of the model has been created and some data entered. Some differences, albeit small, can be observed with regards to the occurrence of verification \& validation modelling topics throughout the six timeline plots. As a result two different routes that the modellers seem to follow can be identified. One route seems to follow the sequence: the whole mod- 
el is created and data entered and then the model is checked for any errors. This is more obvious in the timeline plot of modellers 1, 2, 3 and 6 . Whereas the alternative route consists of building smaller parts of the model, followed by entering the data and then validating the model, and then more parts of the model are added and data entered and then again validated, and so on. This is more obvious in the timeline plots of modellers 4 and 5 . It should be noted that this finding is based on the author's observations and future studies could look into this aspect in more detail to confirm that this is true for other modellers.

Results \& experimentation bars are less frequent as this modelling topic has not been attended to extensively by participating modellers. This topic seems to be attended to at the end of the protocol, with the exception of modeller 3 , who referred to experimentation at the beginning of the protocol, but was unable to attend to it after having first coded the model because the model was not completely finished. Due to this, the timeline plot for modeller 3 was somewhat different compared to that of other modellers. Nevertheless, the protocol was still considered appropriate because the pattern of attention overall fits the pattern of the other five modellers, who still paid little attention to the last two modelling topics. Therefore, this protocol is included in the sample.

Implementation is only attended to by modeller 3, who thought about it at the beginning of the protocol.

\subsection{Pattern of iterations between modelling topics}

The transition of modellers' attention between topics is next considered in order to understand the pattern of iterations followed by the six expert modellers. Transition matrices are developed representing the cross-tabulation of the sequence of attention between successive pairs of episodes in a protocol. Table 3 displays the number of transitions identified for each modeller. These are normalised in proportions, to account for the differing number of episodes identified in each protocol. Out of the six expert modellers, the highest number of transitions is identified for modellers 6,2 and 4 , with proportion of transitions $72 \%, 63 \%$ and $63 \%$ 
respectively. For the rest of the modellers a smaller number of transitions is observed with proportions ranging around 50\%, where modellers 5 and 3 display a slightly lower level of transitions.

Higher transitions have been associated with better and more iterative design processes (Atman et al. 1999; Wang and Brooks 2007; Willemain and Powell 2007). However, this is not the case for the current sample and perhaps very high iteration rates may probably not be related to good modelling practices. The scatter plot in Figure 4 displays the amount of transitions carried out against the level of experience for each modeller. It shows that there is no clear relationship between the amount of transitions carried out and the level of experience for the six modellers. This finding is obtained from a relatively small sample size. A larger sample size could have provided different results. Furthermore, years of experience may not necessarily represent an appropriate measure of the modellers' level of experience, as the latter depends also on how many modelling projects each modeller undertakes. Future studies could consider using a more salient measure such as the number of projects completed by each modeller instead.

\section{TABLE 3 GOES ABOUT HERE}

\section{FIGURE 4 GOES ABOUT HERE}

The individual transition matrices for each modeller (Figure 5) are now considered in more detail. Each cell shows the frequency of iterations between two modelling topics calculated as a proportion of the total number of iterations identified. Using proportions is considered more appropriate instead of the number of transitions, given that the latter varies significantly from one modeller to the other. The cells in each transition matrix have been highlighted according to the number of transitions identified. The darker shades signify a higher number of transitions as per the legend provided at the bottom of Figure 5. These in turn, define the modellers' dominant loop of thinking. 


\section{FIGURE 5 GOES ABOUT HERE}

Turning now the attention to the dominant loop of thinking observed in the individual transition matrices (Figure 5), the six modellers seem to depict different patterns in their loop of thinking depending on whether this displays a wider (modellers 1, 2 and 4) or more focused (modellers 3, 5 and 6) spread. The thinking process of modellers 1, 2 and 4 seems to dominate alternating between model coding and data inputs to a considerably high level for $34 \%(17+17), 45 \%(21+24)$ and $35 \%(17+18)$ of total transitions respectively. In addition, transitions between verification \& validation and model coding are also significant, albeit lower for $22 \%(11+11)$, $20 \%(12+8)$ and $32 \%(16+16)$ of transitions respectively. This implies that modellers 1,2 and 4 iterate more often between the topics of model coding, data inputs and to some extent verification \& validation.

For modellers 3, 5 and 6, their dominant loop of thinking seems to consist of a wider cycle of transitions between modelling topics. Albeit a smaller overall intensity is present (compared to modellers 1,2 and 4) as depicted by the lighter shade (Fig 5), their dominant loop includes relatively more transitions from and to conceptual modelling in addition to model coding, data inputs and verification \& validation. The iterations for modellers 3 and 6 are spread evenly between all the modelling topics in the dominant loop. The dominant loop for modeller 5 depicts a similar shape, however, relatively higher iterations between model coding and verification \& validation are observed compared to modellers 3 and 6 . This is explained as a result of modeller 5 keenness to check the model at regular points during the modelling exercise to ensure that the model built was valid.

From the observed patterns of iterations, inferences can be made about the modelling activity depicted by the expert modellers participating in the task. It is clear that model coding is a central activity to which modellers return often. Similar observations were also made by Willemain (1995) and Wang and Brooks (2008). Furthermore, two different patterns of iterations seem to characterise the experts' modelling activity. Some 
modellers tend to depict a focused dominant loop of thinking due to the concentrated transitions among 2 to 3 modelling topics (model coding, data inputs and verification \& validation), whereas others depict a wider dominant loop of thinking with transitions spread more evenly among the four core topics (conceptual modelling, model coding, data inputs and verification \& validation). Considering the modellers' profile (Table 1 ) and the patterns of iterations observed, no distinguishing characteristics among the two groups of modellers can be identified. However, considering the groupings of modellers based on the radar diagrams with the proportions of attention paid to the four core modelling topics (section 4.1) some level of association can be identified. Two of the modellers ( 3 and 6 ) who depict a wider dominant loop of thinking, were classified as undertaking a balanced style. Whereas most modellers who depict a coding-oriented or reduced conceptualisation style seem to depict a concentration of thinking on specific modelling topics (modellers 1, 2 and 4).

\section{Discussion}

This paper builds on existing experimental research and it provides an exploratory study of the model development processes, focusing more specifically on discrete-event simulation modelling. Six practitioners, expert modellers in DES, with experience between 4 to 13 years were involved. Their modelling activity was observed while at the same time, they 'spoke aloud' their thoughts. This research provides a better understanding of the modelling behaviour practiced by expert DES modellers, with a view to informing existing research and practice in the DES field. It identifies areas for further improvement in the practice of DES modelling, while at the same time the insights gained can in turn be useful for teaching modelling skills to novice modellers. In addition, the methods used in this study provide a contribution to a limited qualitative research area exploring modelling behaviour in OR. A detailed description of the processes followed as well as how verbal protocol analysis was carried out is provided. Researchers wishing to carry out similar studies could find this useful. This section discusses the key findings resulting from this research, considering the implications for 
simulation practice and research. Furthermore considerations about the limitations of this study are also made.

\subsection{Summary of findings}

With regards to the attention paid to the different modelling activities, model coding is the most frequently attended topic that the six expert modellers return to most often. This finding is consistent with the proportions reported in the survey undertaken by Hollocks (2001). However, the proportion of attention paid to the modelling activities differs from the estimates suggested by Shannon (1998) and the proportions reported for the one expert modeller by the Wang and Brooks (2007) study. Indeed, Wang and Brooks (2007) found that the expert modeller, who happened to be an academic, paid the highest amount of attention to conceptual modelling, which is attended to comparatively less by expert modellers in the current study, who were practitioners. While these studies carried out a different means of data collection, the current study found that the expert modellers paid less attention to conceptual modelling and problem structuring. This could be due to the improved user interface in modern simulation software, which can enable conceptual modelling to be carried out implicitly while modelling on the computer. I return to this again later on in the discussion. In addition, the simplified nature of the modelling exercise used, which the modellers read in silence at the beginning of the exercise and hence not included in the analysis, could have affected the limited problem structuring and conceptual modelling activity carried out in the current study. When conceptualising, one considers what should be modelled and how (Robinson 2008). If the problem was clear from reading the case description, then this would result in less attention paid to model objectives and conceptual modelling during the modelling exercise. For larger studies, some formal level of conceptualisation is required, especially if a model concept needs to be agreed first with the client. Hence in real life simulation studies, the amount of attention paid to conceptual modelling may differ depending on the type, purpose and size of models built. 
Another observation was that the equivalent experimentation and post-experimentation activities were attended to significantly less by the expert modellers in this study, compared to all aforementioned studies. This is an unexpected result, but it can however be attributed to the absence of a real life client and to the laboratory nature of the exercise, which did not allow the modellers to spend time on reporting the results or on considering scenarios. While it would be concerning if this finding was true, it is believed that reporting scenario results and preparing reports is an inherent task of OR analysts in real life studies, including simulation projects, which can be presented either in a written or in oral presentations to the client.

In addition, this study looked in more detail at the attention the expert modellers paid to four core modelling activities conceptual modelling, model coding, data inputs and verification \& validation. Three different patterns of modelling behaviour, the model coding-oriented, reduced conceptualisation and balanced style were identified, based on the proportion of time spent on the four activities, albeit this was a tentative finding based on the author's observations of the visual differences in the shape of the different radar diagrams. Considering the modellers' profiles, these patterns of modelling behaviour seem to be down to the individual modeller style. While a larger sample could have allowed for more in-depth analysis, this finding provides useful insights into simulation modelling practice. It can serve as a guide for modellers in their modelling activity by considering which modelling style do they (or should aim to) adopt, the model coding-oriented, the reduced conceptualisation or the balanced style, considering at the same time project priorities.

Considering the sequence of modelling topics during the modelling process, the expert modellers start initially by swapping between conceptual modelling, model coding and data inputs, while later on in the protocol the thinking process progresses to the other modelling topics, such as verification and validation or model results. There seems to be an overall pattern of sequence, which suggests some level of iterations among topics. As expected, all the expert modellers involved in this study, iterate frequently among modelling topics. Due to the small sample size, it was not possible to explore the effect of experience on level of transitions carried out 
by the expert modellers. In his study Willemain's (1995) suggests that more experienced modellers tend to iterate more. Similarly in a study of novice modellers, Willemain and Powell (2007) found that novice modellers exhibit a lower level of iterations. This suggests that iterations come naturally as a result of the experience and modelling practice. Indeed Morris (1967) mentions that with practice, modelling activities become more intuitive which subsequently results in a more holistic approach to the modelling task, that is an ability to switch attention frequently between modelling activities. In educating novice modellers, it would be useful to introduce some level of iteration in their activities by identifying a pattern of links between the different modelling activities during a modelling task. This would in turn result in an improved modelling practice. An implication for further research would be to identify the most effective dominant loops that would lead to a more successful modelling practice.

A detailed analysis of the transition matrices shows that model coding seems to be a central activity during the modelling task. The majority of iterations are identified between conceptual modelling, model coding, data inputs and verification \& validation. These transitions determine the dominant loop in the modellers' thinking process. On average, all participating modellers alternate mainly between conceptual modelling and model coding and the opposite (for an average of $17 \%$ of the iterations), between model coding and data inputs and the opposite (for an average of $27 \%$ of iterations) and between model coding and verification $\&$ validation and the opposite (for an average $22 \%$ of iterations). It is not possible to establish whether this is a result of the case study used in this study. Future research could consider using different case studies and compare the results.

Furthermore, the pattern of transitions between modelling activities for each individual modeller suggests that the modellers choose between two main styles, the focused and the wider dominant loop of thinking. Some modellers concentrate their attention among 2-3 modelling topics, while others spread their attention more evenly and widely among 4 modelling topics. These styles seem to be loosely related to the modelling styles identified for the attention paid among the four main topics (section 4.1). 
Returning to the high number of transitions observed between conceptual modelling and model coding topics, albeit not the highest, it suggests that most expert modellers undertook conceptual modelling alongside model coding. This shows that, due to their experience, expert modellers tend to abstract the problem situation into a model and devise relevant modelling strategies inherently while modelling. In addition, some bars in the timeline plots were considered to refer to both topics (model coding and conceptual modelling) and hence shared half and half between them. This could be explained due to the fact that the expert modellers were confident with modelling at the same time as conceptualizing, but also the "easier to use" simulation software makes it possible for one to conceptualize at the same time as modelling on the computer. However, this is not recommended for novice modellers with little experience. Future research could consider undertaking a more in-depth analysis of experts' conceptual modelling processes with the view to identifying efficient modes of conceptual modelling practice, which can be taught to novice modellers.

\subsection{Study limitations}

After having discussed the findings of this study, the limitations, referring to the data collected and methods employed, are now considered. First and foremost, it should be noted that the analysis of the protocols is based on the researcher's interpretation of participants' verbalizations. Hence, some level of subjectivity is present in the results of the study. To minimize the effect of subjectivity, the protocols were coded 3 times, involving in one case a third party. This study has an exploratory nature and it does not claim to provide generalizable findings. A bigger sample size could have also provided more representative results, however, at the time of data collection these were the main UK contacts available to the researcher. Due to project timescales it was not feasible to obtain a larger sample. For future research, a larger sample size and alternate case studies could be used with the view to statistically confirm the results and the modelling styles identified in the current study. Furthermore, a wider sample base, involving expert modellers from different countries, US, Europe, etc. could be used to consider whether different patterns of modelling behaviour are present. 
With reference to the data collection process (verbal protocol analysis), the modellers were asked to speak aloud their thoughts while modelling. It is obviously considered difficult to think and speak at the same time (Ericsson and Simon 1984) and modellers did not have prior experience of this type of procedure. In order to help modellers to think aloud their thoughts practice exercises were used before each session. None of the modellers encountered any problems during the practice sessions and by the end they seemed to become more fluent.

In addition, the modelling sessions were implemented as part of controlled laboratory settings, where the modellers at times felt the pressure of time or the pressure of being observed. The task given to the participants was a simple and a quite structured task to ensure completion of the exercise for a limited amount of time. This has to some extent affected the smaller amount of verbalisations for modelling topics such as: problem structuring, results \& experimentation and implementation. Furthermore the absence of a real life client has to some extent affected the amount of attention the modellers paid to reporting the results and the implementation of findings.

\section{Conclusions}

This study takes an empirical approach to exploring the model development process in discrete-event simulation as followed by expert modellers in the field. The modelling process is analysed by undertaking a quantitative analysis of the thought processes, based on the modelling activities or topics, that expert modellers think about during a modelling exercise. The findings of this study are considered relevant to the existing research and practice in simulation modelling as well as to education. The knowledge gained opens up avenues for further consideration with regards to the practical application of DES in relation to the following three areas:

1) Design of the graphical user interface (GUI) in DES software. Having found that expert modellers switch often between modelling topics, one further consideration would be to identify how the GUI in 
existing software may help or hinder this activity. Future work could carry out experiments to explore how the "switching between topics" behaviour is related to variations in GUI design.

2) Education of novice modellers. The research presented in this study unveils the expert modellers' behaviour(s), which are examples for novices in simulation to 'mimic'. This leads to pedagogical considerations in education about how should novice modellers be taught in a way that encourages a replication of experts' behaviours. For example, engaging students with multiple instances of similar exercises could be included in students' educational curriculum. Repeated exposure to the mental activity required to build DES models under the supervision of an experienced modeller could be a useful means of teaching modelling skills to novices. It would also help students gain the ability to build simulation models in one hour, which can be considered a useful skill.

3) The link between modelling styles and effectiveness of modelling. This study puts forward modelling styles based on the topics modellers concentrate their modelling activity on and on their 'switching between topics' behaviour, which has not been addressed so far by the existing literature. While this study was not set out to evaluate the effectiveness of modelling, future research could consider the link between these, which could lead to identifying 'more effective' styles leading to better quality models.

The findings of the current study could be supplemented by a qualitative study of the verbal protocols which would carry out an in-depth analysis of the activities carried out by the modellers during the modelling exercise and the resulting outcomes and changes to the models obtained. Furthermore, modellers undertaking real projects could be followed. A similar study could be undertaken to follow modellers using other modelling approaches, such as system dynamics and agent-based modelling. Researchers wishing to carry out a similar 
line of research should consider issues related to subjectivity which in turn can affect the generalizability of the findings.

\section{ACKNOWLEDGMENTS}

The author would like to thank Professor Stewart Robinson for the help and suggestions provided. Thanks also go to the modellers who participated in the experiment and to Suchi Collingwood for the help in blind checking the protocols. This paper is an extended version of the paper titled: "Model development in discrete-event simulation: Insights from six expert modellers", presented at the 2011 Winter Simulation Conference.

\section{References}

Atman, C. J., J. R. Chimka, K. M. Bursic and H. L. Nachtmann (1999). "A comparison of freshman and senior engineering design processes." Design Studies 20(2): 131-152.

Balci, O. (1994). "Validation, verification, and testing techniques throughout the life cycle of a simulation study." Annals of Operations Research 53(1): 121-173.

Balci, O. (2012). "A life cycle for modeling and simulation." Simulation: Transactions of the Society for Modeling and Simulation International advance online publication 24 February 2012(doi: 10.1177/0037549712438469).

Balci, O. and R. E. Nance (1987). "Simulation Model Development Environments: A Research Prototype." The Journal of the Operational Research Society 38(8): 753-763.

Banks, J., J. S. CarsonII, B. L. Nelson and D. M. Micol (2001). Discrete-Event System Simulation. Prentice Hall: Upper Saddle River, New Jersey.

Cochran, J. K., G. T. Mackulak and P. A. Savory (1995). "Simulation Project Characteristics in Industrial Settings." Interfaces 25(4): 104-113.

Ericsson, K. A. and H. A. Simon (1984). Protocol Analysis: Verbal Reports as Data, The MIT Press.

Foss, B. A., B. Lohmann and W. Marquardt (1998). "A field study of the industrial modeling process." Journal of Process Control 8(5): 325-338.

Grove, P., J. MacLeod and D. Godfrey (1998). "Forecasting the prison population." OR Insight 11(1).

Hollocks, B. (2001). "Discrete-event Simulation: An enquiry into user practice." Simulation Practice and Theory 8: 451471.

Law, A. M. (2007). Simulation modeling and analysis. McGraw-Hill: Boston; London.

Morris, W. T. (1967). "On the art of modeling." Management Science 12(12): B707-B717. 
Nance, R. (1994). "The Conical Methodology and the evolution of simulation model development." Annals of Operations Research 53(1): 1-45.

Nance, R. E. and R. G. Sargent (2002). "Perspectives on the Evolution of Simulation." Operations Research 50(1): 161172.

Pidd, M. (1999). "Just Modeling Through: A Rough Guide to Modeling." INTERFACES 29(2): 118-132.

Pidd, M. (2004). Computer Simulation in Management Science. John Wiley \& Sons: Chichester.

Powell, S. G. and T. R. Willemain (2007). "How novices formulate models. Part I: qualitative insights and implications for teaching." Journal of the Operational Research Society 58(8): 983-995.

Robinson, S. (2004). Simulation: The Practice of Model Development and Use. John Wiley \& Sons: Chichester.

Robinson, S. (2005). "Discrete-event simulation: from the pioneers to the present, what next?" Journal of the Operational Research Society 56(6): 619-629.

Robinson, S. (2008). "Conceptual modelling for simulation Part I: Definition and requirements." Journal of the Operational Research Society 59(3): 278-290.

Sanchez, P. J. (2007). Fundamentals of simulation modeling. Proceedings of the 39th conference on Winter simulation: 40 years! The best is yet to come. S. G. Henderson, B. Biller, M.-H. Hsieh, J. Shortle, J. D. Tew and R. R. Barton. Washington D.C., IEEE Press: 54-62.

Sargent, R. G. (2010). Verification and Validation of Simulation Models. Proceedings of the 2010 Winter Simulation Conference. S. J. B. Johansson, J. Montoya-Torres, J. Hugan, E. Yucesan (eds.). Baltimore, Maryland, IEEE: pp. 166-188.

Sargent, R. G., R. E. Nance, C. M. Overstreet, S. Robinson and J. Talbot (2006). The simulation project life-cycle: models and realities. In the proceedings of the 38th Winter Simulation Conference. F. P. W. L. F. Perrone, J. Liu, B. G. Lawson, D. M. Nicol, and R. M. Fujimoto, (eds.), Winter Simulation Conference: pp. 863-871.

Shannon, R. E. (1975). Systems Simulation: The Art and Science. Prentice Hall: Englewood Cliffs, NJ.

Shannon, R. E. (1998). Introduction to the art and science of simulation. Proceedings of the 30th conference on Winter simulation. E. F. W. D.J. Medeiros, J.S. Carson and M.S. Manivannan, (eds.). Washington, D.C., United States, IEEE Computer Society Press: pp. 7-14.

Tako, A. A. (2009). Development and use of simulation models in Operational Research: a comparison of discrete-event simulation and system dynamics. Warwick Business School. Coventry, University of Warwick. PhD thesis.

Tako, A. A. and S. Robinson (2009). "Comparing discrete-event simulation and system dynamics: Users' perceptions." Journal of the Operational Research Society 60(3): 296-312.

Tako, A. A. and S. Robinson (2010). "Model development in discrete-event simulation and system dynamics: an empirical view." European Journal of Operational Research 207: 784-794.

Todd, P. and I. Benbasat (1987). "Process tracing methods in decision support systems research: Exploring the black box." MIS Quarterly 11(4): 493-512.

Waisel, L. B., W. A. Wallace and T. R. Willemain (2008). "Visualization and model formulation: an analysis of the sketches of expert modellers." J Oper Res Soc 59(3): 353-361. 
Wang, W. and R. J. Brooks (2007). "Improving the understanding of conceptual modelling." Journal of Simulation 1(3): 153-158.

Willemain, T. R. (1994). "Insights on modeling from a dozen experts." Operations Research 42(2): 213-222.

Willemain, T. R. (1995). "Model formulation: What experts think about and when." Operations Research 43(6): 916-932.

Willemain, T. R. and S. G. Powell (2007). "How novices formulate models. Part II: a quantitative description of behaviour." J Oper Res Soc 58(10): 1271-1283.

\section{List of tables}

Table 1: Profiles of participating expert modellers

\begin{tabular}{|c|r|l|l|l|}
\hline Modeller & $\begin{array}{l}\text { Modelling } \\
\text { experience }\end{array}$ & $\begin{array}{l}\text { Simulation } \\
\text { software }\end{array}$ & $\begin{array}{l}\text { Source of obtaining } \\
\text { simulation skills }\end{array}$ & Education Level \& degree title \\
\hline 1 & 9 years & Witness & Job & BEng Manufacturing Engineering \\
\hline 2 & 4 years & Simul8 & Studies AND Job & MSc in Operational Research \\
\hline 3 & 13 years & Flexsim & Job & BSc in Computer Science \\
\hline 4 & 8 years & Simul8 & Job & MSc in Engineering \\
\hline 5 & 4 years & Witness & Studies AND Job & $\begin{array}{l}\text { MSc in Applied Statistics \& } \\
\text { Management Science }\end{array}$ \\
\hline 6 & 8 years & Witness & Studies AND Job & BSc in Industrial Design \\
\hline
\end{tabular}

Table 2: Comparing the proportion of attention paid to modelling topics by the six expert modellers in the current study against the one expert in Wang and Brooks' (2007) study.

Modelling topic

Problem structuring

Conceptual Modelling

Data Inputs

Model Coding

Verification \& Validation

Experimentation

Implementation/Report writing

$\begin{array}{ccc}\text { Current study } & \text { Wang and Brooks study } & \text { Differences } \\ 1 \% & 13 \% & -12 \% \\ 13 \% & 23 \% & -10 \% \\ 16 \% & 14 \% & 2 \% \\ 42 \% & 18 \% & 24 \% \\ 20 \% & 19 \% & 1 \% \\ 5 \% & 8 \% & -3 \% \\ 0 \% & 6 \% & -6 \%\end{array}$


Table 3: Number of transitions of attention and the equivalent proportions of transitions calculated by dividing the number of transitions by the total number of episodes identified in each protocol.

\begin{tabular}{|c|c|c|c|}
\hline Modeller & Number of transitions & Total episodes & Proportion of transitions \\
\hline 1 & 66 & 119 & $55 \%$ \\
\hline 2 & 85 & 135 & $63 \%$ \\
\hline 3 & 136 & 297 & $46 \%$ \\
\hline 4 & 77 & 122 & $63 \%$ \\
\hline 5 & 141 & 286 & $49 \%$ \\
\hline 6 & 78 & 109 & $72 \%$ \\
\hline
\end{tabular}

\section{List of figures}

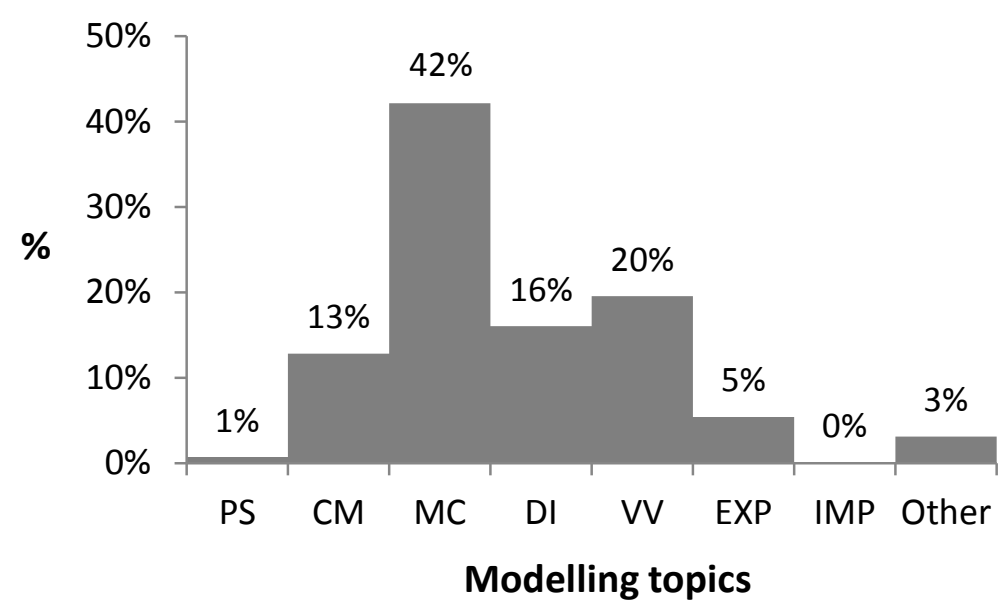

Figure 1: Proportion of attention paid by modelling topic by the six modellers 

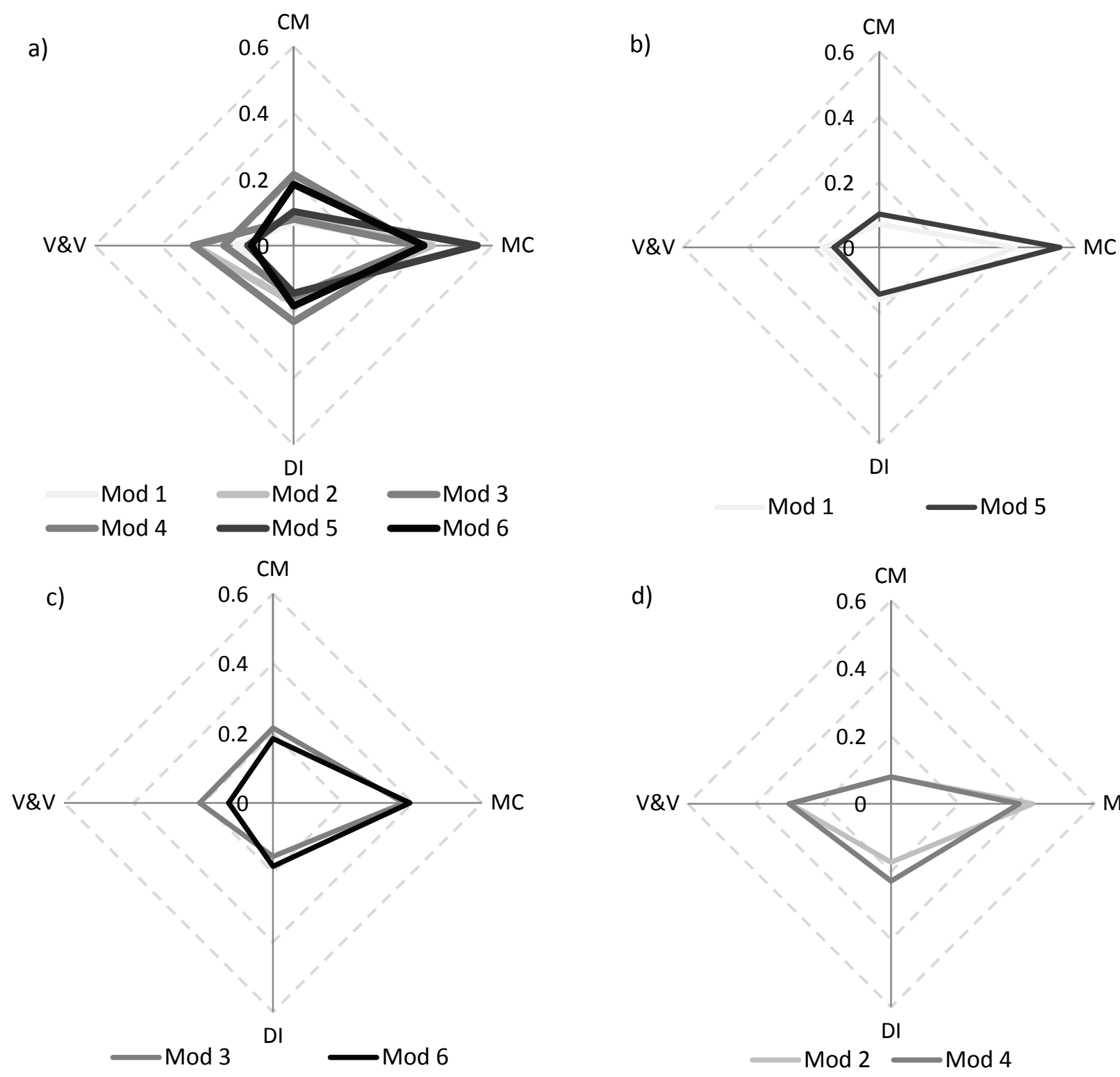

DI

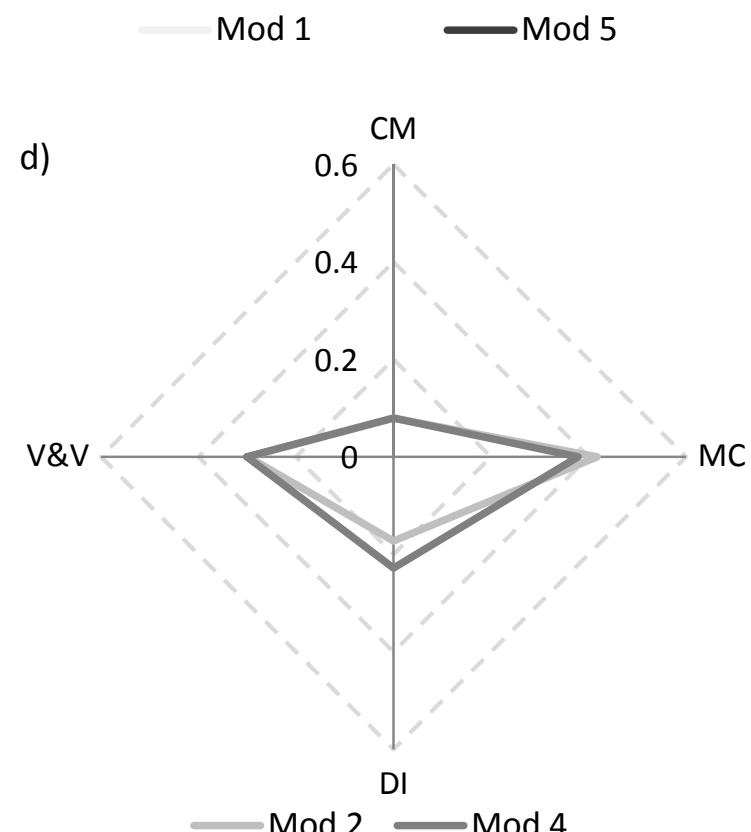

Figure 2: Radar diagrams displaying the proportion of words verbalized between the four most common modelling topics (Conceptual modelling (CM), Model coding (MC), Data inputs (DI) and Verification \& Validation (V\&V) by modeller. Diagram a) displays data for all six modellers, whereas diagrams with similar shapes are displayed separately in b), c) and d). 

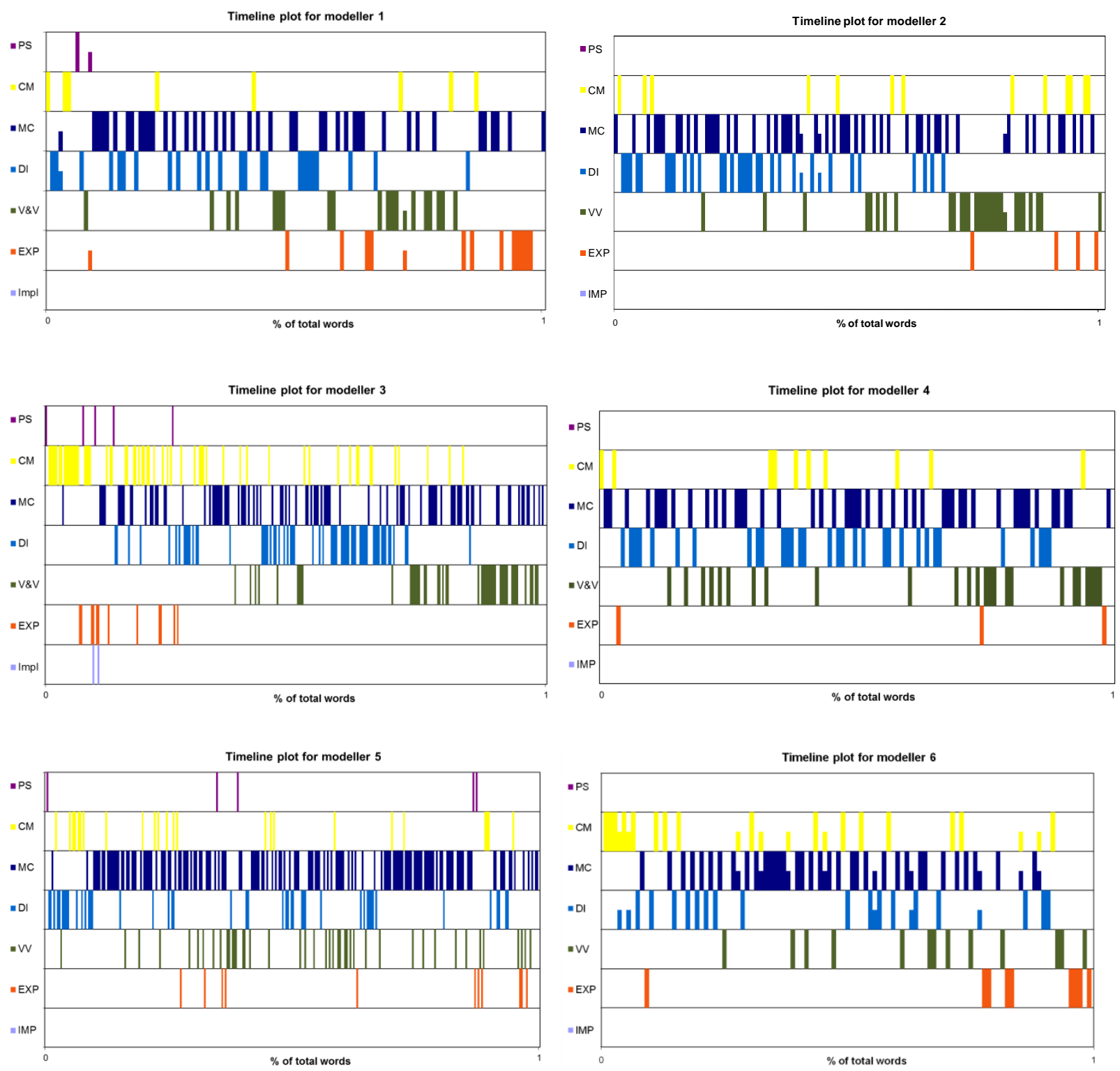

Figure 3: Timeline plots for the six expert modellers 


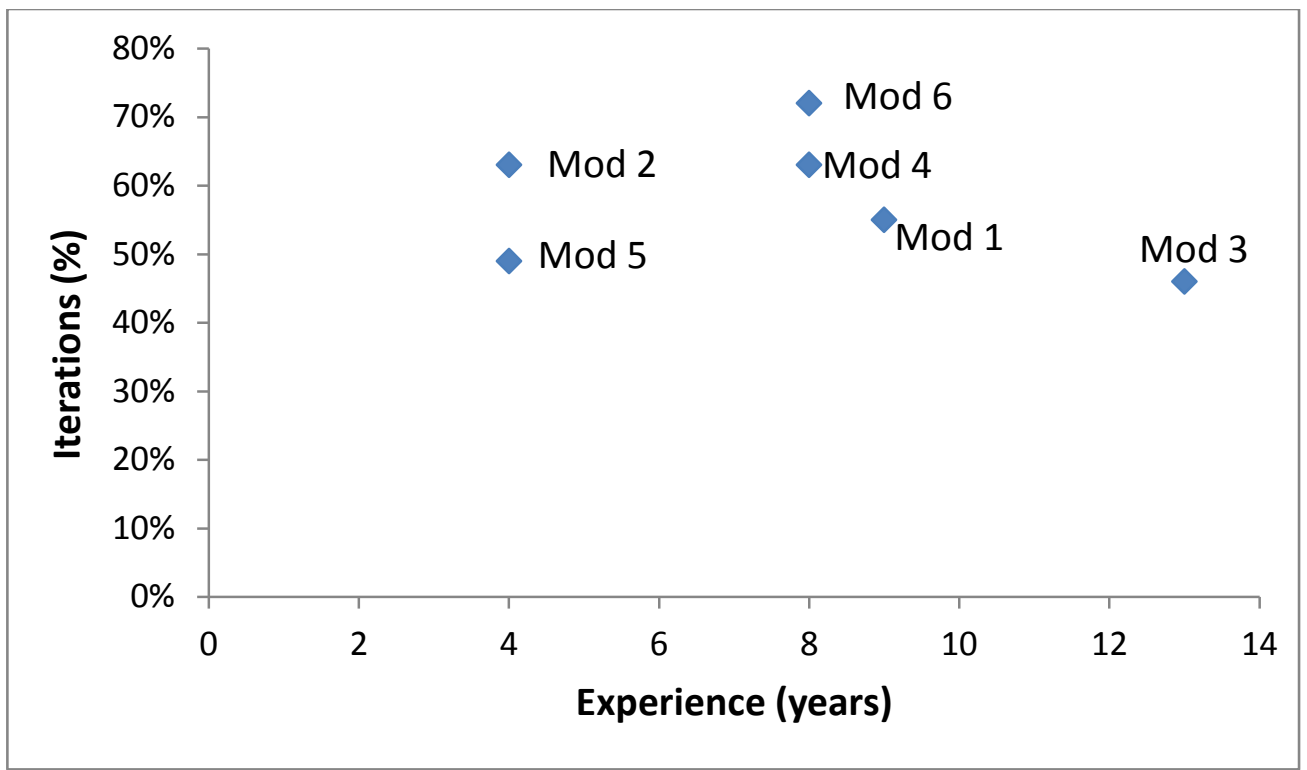

Figure 4: Scatter plot of modellers' level of transitions and experience length 
Modeller 1

\begin{tabular}{l|rrrrrrr}
\hline & PS & CM & MC & \multicolumn{1}{l}{ DI } & VV & RE & IMPL \\
\hline PS & - & & 0.02 & 0.02 & & & \\
CM & & - & 0.03 & 0.02 & 0.03 & 0.02 & \\
MC & & 0.05 & - & 0.17 & 0.11 & 0.06 & \\
DI & & 0.02 & 0.17 & - & 0.03 & 0.02 & \\
VV & 0.02 & 0.02 & 0.11 & 0.02 & - & 0.03 & \\
RE & & 0.02 & 0.05 & 0.03 & & - & \\
IMPL & & & & & & & - \\
\hline
\end{tabular}

Modeller 3

\begin{tabular}{lrrrrrrr}
\hline & PS & CM & MC & \multicolumn{1}{l}{ DI } & VV & RE & IMPL \\
\hline PS & - & 0.01 & & 0.01 & & 0.01 & \\
CM & 0.01 & - & 0.13 & 0.06 & & 0.03 & \\
MC & & 0.11 & - & 0.12 & 0.10 & 0.01 & \\
DI & & 0.07 & 0.11 & - & 0.01 & 0.01 & \\
VV & & 0.02 & 0.08 & 0.01 & - & & \\
RE & 0.01 & 0.02 & 0.01 & 0.01 & & - & 0.01 \\
IMPL & 0.01 & & 0.01 & & & & - \\
\hline
\end{tabular}

Modeller 5

\begin{tabular}{lrrrrrrrr}
\hline & PS & CM & MC & DI & VV & RE & IMPL \\
\hline PS & - & & 0.01 & 0.01 & & 0.01 & \\
CM & & - & 0.09 & 0.04 & 0.01 & & \\
MC & 0.01 & 0.07 & - & 0.10 & 0.16 & 0.02 & \\
DI & & 0.04 & 0.09 & - & 0.04 & & \\
VV & 0.01 & 0.02 & 0.13 & 0.04 & - & 0.04 & \\
RE & 0.01 & & 0.04 & & 0.03 & - & \\
IMPL & & & & & & & - \\
\hline
\end{tabular}

Modeller 2

\begin{tabular}{|c|c|c|c|c|c|c|c|}
\hline & PS & CM & MC & DI & VV & RE & IMPL \\
\hline PS & - & & & & & & \\
\hline $\mathrm{CM}$ & & - & 0.08 & 0.02 & 0.02 & & \\
\hline MC & & 0.07 & - & 0.24 & 0.08 & 0.02 & \\
\hline DI & & 0.01 & 0.21 & - & 0.04 & & \\
\hline VV & & 0.02 & 0.12 & & - & 0.01 & \\
\hline RE & & & 0.02 & & 0.02 & - & \\
\hline \multirow[t]{3}{*}{ IMPL } & & & & & & & - \\
\hline & \multicolumn{6}{|c|}{ Modeller 4} & \\
\hline & PS & CM & MC & DI & V\&V & R\&E & IMPL \\
\hline PS & - & & & & & & \\
\hline CM & & - & 0.04 & 0.05 & 0.01 & 0.01 & \\
\hline MC & & 0.04 & - & 0.18 & 0.16 & & \\
\hline DI & & 0.04 & 0.17 & - & 0.04 & & \\
\hline V\&V & & 0.03 & 0.16 & 0.01 & - & 0.03 & \\
\hline R\&E & & & 0.01 & 0.01 & 0.01 & - & \\
\hline IMPL & & & & & & & - \\
\hline
\end{tabular}

\begin{tabular}{lrrrrrrrr}
\multicolumn{7}{c}{ Modeller 6 } \\
\hline & PS & CM & MC & DI & V\&V & R\&E & IMPL \\
\hline PS & - & & & & & & \\
CM & & - & 0.12 & 0.10 & 0.03 & & \\
MC & & 0.13 & - & 0.14 & 0.010 & 0.01 & \\
DI & & 0.06 & 0.15 & - & & 0.01 & \\
VV & & 0.01 & 0.06 & 0.01 & - & 0.03 & \\
R\&E & & & & 0.01 & 0.01 & - & \\
IMPL & & & & & & - \\
\hline
\end{tabular}

\section{Legend}

$$
0 \quad 0.01-0.05 \quad 0.06-0.10 \quad 0.11-0.15 \quad 0.16+
$$

Figure 5: Individual transition matrices for the six expert modellers representing the percentage of transitions between modelling topics as a proportion of the total number of transitions (empty cells represent 0 ). 